Cahiers $d u$ MONDE RUSSE

\section{Cahiers du monde russe}

Russie - Empire russe - Union soviétique et États indépendants

$49 / 4 \mid 2008$

Destins individuels et terreur. Jeunesse dans la société post-stalinienne

\title{
Angela Rustemeyer, Dissens und Ehre
}

\section{Malte Griesse}

\section{(2) OpenEdition}

Journals

Édition électronique

URL : https://journals.openedition.org/monderusse/6910

DOI : 10.4000/monderusse.6910

ISSN : $1777-5388$

Éditeur

Éditions de l'EHESS

Édition imprimée

Date de publication : 28 décembre 2008

Pagination : 705-710

ISBN : 978-2-7132-2197-2

ISSN : $1252-6576$

Référence électronique

Malte Griesse, "Angela Rustemeyer, Dissens und Ehre », Cahiers du monde russe [En ligne], 49/4 I 2008, mis en ligne le 23 décembre 2009, consulté le 03 septembre 2022. URL : http://

journals.openedition.org/monderusse/6910; DOI : https://doi.org/10.4000/monderusse.6910

Ce document a été généré automatiquement le 3 septembre 2022

Tous droits réservés 


\title{
Angela Rustemeyer, Dissens und Ehre
}

\author{
Malte Griesse
}

\section{RÉFÉRENCE}

Angela RUSTEMEYER, Dissens und Ehre. Majestätsverbrechen in Rußland (1600-1800). Wiesbaden : Harrassowitz Verlag, 2006, X +462 p. (Forschungen zur osteuropäischen Geschichte 69).

1 Dans son livre Dissidence et honneur, Angela Rustemeyer brosse un vaste panorama des actions et des comportements sanctionnés comme crimes de lèse-majesté à l'époque moderne. Refusant de se limiter à la Russie, comme le titre pourrait le suggérer, elle place le phénomène dans un contexte européen plus large. Ce cadre est loin d'être purement nominal : non seulement l'auteur fait le point sur l'état actuel de la recherche en ce qui concerne les pays d'Europe centrale et occidentale, mais elle a en outre mené ses propres recherches sur des terrains variés, y compris dans les archives, celles notamment de l'Empire des Habsbourgs. Le centre d'intérêt de sa monographie n'en demeure pas moins la Russie, où Rustemeyer s'est appuyée essentiellement sur les procès de lèse-majesté, littéralement : pour " paroles et actes concernant le souverain » (slovo $i$ delo gosudarevy). Cette catégorie regroupe tout un éventail de délits considérés comme crimes de lèse-majesté et dont l'âge d'or (si l'on peut dire) se place aux XVII et $\mathrm{XVIII}^{\mathrm{e}}$ siècles, d'où le cadre chronologique de cette étude. Si au XVII siècle les sources sont encore dispersées entre plusieurs institutions, ce qui rend difficile l'analyse quantitative, les réformes de Pierre $\mathrm{I}^{\text {er }}$ commencent par centraliser les affaires de slovo $i$ delo entre les mains du secrétariat de Preobražensk(Preobraženskij prikaz, à partir de 1702) puis les confient à la Chancellerie secrète (Tajnaja kanceljarija, à partir de 1718 et jusqu'en 1731). Finalement, à partir du règne de Catherine II, c'est l'Expédition secrète (Tajnaja ekspedicija) qui en est chargée jusqu'en 1801, lorsque le crime de lèse-majesté lui-même 
est aboli (pour réapparaître sous une autre forme à la suite du soulèvement des décembristes).

2 Méthodologiquement, Rustemeyer s'appuie sur les approches relativement récentes des études de la criminalité qui cherchent à conjuguer une histoire sociale et culturelle d'en bas avec celle du pouvoir et de ses démarches pour assurer et systématiser le contrôle social de ses sujets. Elle s'interroge ainsi sur la transition du modèle du Personenverbandsstaat ${ }^{1}$ médiéval vers un rapport privilégié institutionnalisé entre souverain et sujets, rapport caractéristique pour la constitution de la monarchie absolue comme base de l'État moderne ${ }^{2}$. Elle s'intéresse aux interactions concrètes entre des individus relevant de groupes sociaux variés, les institutions et autorités régionales et centrales et enfin le monarque, interactions qui comprennent des prises de position, des remarques ou plus simplement des injures au sujet de la politique, mais aussi des dénonciations et des poursuites judiciaires où l'on voit transparaître les intérêts et les catégories de jugement des différents acteurs. Alors que nos idées sur la vie quotidienne et les mentalités des couches sociales inférieures à l'époque moderne viennent largement de l'image qu'en donnent les autorités, les administrés eux-mêmes restant généralement muets dans les sources, les procès pour slovo $i$ delo donnent la parole à toutes les strates de la population, y compris à celle, largement majoritaire, des paysans, bien que là encore les illettrés restent proportionnellement sous-représentés.

3 Pour le XVIII ${ }^{\mathrm{e}}$ siècle, Rustemeyer estime à 10 000-12 000 le nombre des cas de lèse-majesté instruits au niveau de l'autorité centrale, ce qui est minime par rapport à l'ensemble des délits ayant fait l'objet de poursuites, mais considérable par rapport aux cas de lèsemajesté dans d'autres pays européens. Faut-il y voir un symptôme révélateur du despotisme autocratique, du règne de l'arbitraire qui sépare la Russie du reste de l'Europe, comme le veut le cliché habituel, déjà diffusé dans les écrits de nombreux étrangers de l'époque? Sans nier la brutalité des châtiments pratiqués en Russie, où l'on n'hésite pas à mutiler les condamnés, Rustemeyer prend avec véhémence le contre-pied de ce stéréotype, qui prétend établir une ligne de démarcation entre civilisation et barbarie, ou entre État de droit (en Europe, bien sûr) et autocratie arbitraire (en Russie). Le caractère comparatif de l'ouvrage permet de balayer certaines idées reçues et de remettre dans son contexte, par exemple, le recours universel à la torture : à l'époque moderne, elle était employée pour la recherche de la vérité aussi bien en Russie que dans le reste de l'Europe, et continua à l'être, pour les crimes de lèse-majesté, en plein âge des Lumières. Il sert aussi à montrer que la sorcellerie était poursuivie ailleurs avec beaucoup plus de férocité et à une plus grande échelle qu'en Russie. Rustemeyer met ainsi en évidence des évolutions et des processus d'influences réciproques transnationaux qui intègrent, ou plutôt réintègrent, la Russie dans une histoire de l'Europe.

4 C'est le cas par exemple du traitement juridique du crime de lèse-majesté. Elle récuse à la fois la conception nettement péjorative qui nie toute trace du droit romain en Russie, et les histoires apologétiques nationales qui affirment avec fierté le développement autonome du droit russe et de ses pratiques pénales qui se distingueraient par leur humanisme relatif (Rogov). Elle reconstitue avec finesse les liens ténus qui unissent le droit russe ancien aux systèmes de droit européens et byzantin, et notamment l'impact du Code [Statut] polono-lituanien de la fin du Xvi ${ }^{e}$ siècle sur l'Établissement, ou code, de 1649 (Sobornoe uloženie), la première grande compilation ${ }^{3}$ de droit écrit en Russie encore largement appliquée jusqu'au XIX ${ }^{e}$ siècle. Le concept même du crime de lèse-majesté a 
été repris de la Pologne-Lituanie, entre autres, semble-t-il, pour adapter les institutions russes à l'image que donnaient d'elles-mêmes les monarchies absolues naissantes d'Europe, ce qui montre clairement que la filiation des emprunts juridiques remonte bien au-delà du modèle immédiat.

5 Ces emprunts s'inscrivent dans une évolution du procès contradictoire vers un procès inquisitorial, où le dénonciateur du crime n'est plus forcément la victime et qui ouvre la porte à la dénonciation au nom du souverain, ce qui va de concert avec un processus de "verticalisation» du droit à l'échelle européenne. Un comparatisme statique, qui ne tient pas compte des dynamiques évolutives, expliquerait la plus grande souplesse $d u$ droit pénal russe par un moindre degré d'avancement du pays et la survivance d'éléments médiévaux. Mais Rustemeyer ne se satisfait pas de l'hypothèse simplificatrice de l'« arriération» de la Russie, qui ne ferait qu'emprunter ses institutions (avec grand retard) à ses voisins occidentaux plus développés. Les influences transnationales sont réciproques, ce qui apparaît en tout premier lieu dans la répression des transfuges, où l'évolution des cadres judiciaires et des pratiques punitives est intrinsèquement liée aux relations bilatérales avec les pays voisins - processus particulièrement bien documenté pour la Pologne - Lituanie, moins bien pour la Suède et l'Empire ottoman où la base de sources est plus maigre.

6 Rustemeyer répertorie trois délits majeurs qui sont poursuivis de façon privilégiée sous la catégorie des slovo $i$ delo gosudarevy: fuite du pays, révolte et "propos inconvenants " (nepristojnye slova ou reči). Ces derniers ne se limitent pas à la critique du tsar ou de la politique de l'État. Même si les jugements négatifs sur le gouvernement l'emportent parmi les cas relevés, il y a aussi des discours politiques neutres ou même positifs qui font l'objet de poursuites pour ingérence dans la sphère réservée du souverain. Les soulèvements populaires ayant déjà beaucoup attiré l'attention des chercheurs, Rustemeyer se concentre sur leur traitement par la justice, les procédures d'amnistie et la commémoration de l'événement, qui est réservée à l'Église: l'anathème prononcé contre les meneurs est réitéré à travers les siècles, mais on n'érige pratiquement pas de monuments d'infamie « séculaires » comme cela se faisait ailleurs.

7 L'examen des "propos inconvenants» nous donne un aperçu des représentations plus quotidiennes du pouvoir. Le fait même que ce genre de discours fût passible de poursuites est souvent cité comme preuve du caractère proto-totalitaire de l'État moscovite et surtout pétersbourgeois, une interprétation qui apparaît comme peu convaincante au vu du contenu des procès. Pour que les autorités puissent exercer le contrôle social efficace inséparable du totalitarisme, il faut que la population soit prête à coopérer, c'est-à-dire à dénoncer ses voisins. Le nombre de cas relevés montre déjà les limites de la délation, mais leur répartition inégale entre les différentes couches sociales est plus intéressante encore. Alors que les militaires sont les mieux représentés, ce qui s'explique par le lien étroit avec les affaires du souverain que leur impose le service, la majorité écrasante de la population - les paysans - reste très réticente. On trouve des litiges entre ressortissants de différents villages, autour de questions de recrutement et de levée d'impôts, c'est-à-dire des cas où il s'agit de toute façon d'exigences de l'État. Mais les dénonciations au sein d'un même village (derevnja) sont extrêmement rares. Cela vaut aussi pour les représentants du clergé paroissial. Ils figurent en bonne place parmi les dénonciateurs (et les dénoncés), mais généralement il s'agit d'affaires internes à l'Église, qui peuvent être interprétées comme une tentative de serrer les rangs des communautés orthodoxes locales contre la tentation de la vieille foi. Un prêtre de 
village ne dénonce presque jamais ses ouailles, encore moins porte-t-il atteinte au secret de la confession, comme l'aurait voulu Pierre le Grand si le confesseur soupçonnait chez le fidèle de mauvaises intentions contre le monarque. La communauté villageoise se présente ainsi comme un microcosme de dépendances et de solidarités réciproques, où les conflits se réglaient de façon autonome et où la loyauté à l'égard de l'État ou du souverain n'avait que peu de signification. Si l'objectif des procès pour slovo $i$ delo était de faire pénétrer l'État dans la vie quotidienne du village, le résultat serait plutôt un échec.

En ce qui concerne la noblesse, la situation est particulièrement complexe. Second pilier de l'autocratie après le clergé, du moins en principe, celle-ci était censée mettre en place un contrôle social efficace. Mais, en même temps que serviteurs de l'État, les nobles étaient les détenteurs du pouvoir seigneurial et cherchaient à protéger leur domaine des interventions de l'autocratie. Certes, ils ont su tourner à leur avantage la criminalisation du délit de fuite à l'étranger et à partir du moment où ils ont pratiquement cessé de participer à des soulèvements (c'est-à-dire après les révoltes urbaines du milieu du XVII siècle et l'entrée en vigueur de l'Uloženie), le concours massif apporté à la répression leur a attiré la plus grande partie des récompenses distribuées par le tsar.

En ce qui concerne les injures verbales, toutefois, leur rôle est beaucoup plus ambigu. D'une part, experts en chicane, les nobles se servaient du slovo $i$ delo pour gagner leurs conflits d'honneur : puisqu'ils étaient des "gens du tsar ", les insultes que leur lançaient leurs adversaires (à titre purement privé) pouvaient, avec un peu d'adresse, être interprétés comme visant le tsar à travers eux, donc comme des crimes de lèse-majesté, ce qui permettait de leur intenter un procès. Cette stratégie, souvent couronnée de succès, était également pratiquée par des roturiers. D'autre part se montraient peu enclins à accepter que les poursuites touchent leur propre domaine, surtout lorsque les domestiques découvrirent que la dénonciation d'un crime de lèse-majesté était privilégiée, dans la mesure où personne ne pouvait l'étouffer sous peine de se rendre complice du crime; elle constituait donc une arme puissante et ils commencèrent à leur tour à porter plainte. Les procès intentés par des domestiques ou des paysans contre leurs maîtres ou leurs seigneurs n'avaient presque jamais de succès et entraînaient plutôt la punition des dénonciateurs, mais cela ne les empêchait pas de porter plainte : plus qu'une amélioration de leur condition matérielle ils cherchaient à porter atteinte à l'honneur de leur maître. Souvent ces dénonciateurs d'humble condition demandaient à être transférés, avec leur maître, devant l'administration compétente (presque toujours à Moscou ou à Saint-Péterbourg), ce qui, indépendamment de l'issue du procès, représentait pour un noble le comble de l'humiliation.

10 Rustemeyer attribue donc l'abolition, sous Pierre III, de la plainte privilégiée pour « propos inconvenants » à l'influence de la noblesse ainsi qu'à l'effet contre-productif de la criminalisation de ces discours que les représentants de la monarchie ont de plus en plus tendance à qualifier d'inoffensifs. À l'âge des Lumières on peut ainsi observer, sous Catherine II, une « psychologisation » de l'enquête qui va de pair avec l'abandon de la torture comme moyen d'établir la vérité. La tâche consistant à distinguer le bavardage insignifiant de la «foule » des rares cas de vraie subversion est désormais confiée aux gouverneurs nobles (les voevody) qui représentent sur place le monarque. Ils deviennent par conséquent de plus en plus sensibles aux gestes, à l'expression du visage des dénonciateurs comme des dénoncés - un signe indéniable d'individuation, aussi bien de l'enquêteur considéré désormais comme un être raisonnable capable de juger, que du 
criminel présumé qui n'est plus jugé uniquement sur ses mots, mais aussi sur ses intentions. L'image qui en ressort - celle d'une autocratie plutôt souple, ne serait-ce que par la force des circonstances, à la recherche de compromis avec les élites du pays qui, elles, savent parfaitement défendre leurs intérêts - serait symptomatique du caractère consensuel du régime politique de la Russie à l'époque moderne. À en croire Rustemeyer, ce n'est qu'après les guerres napoléoniennes et avec l'arrivée au pouvoir de Nicolas I ${ }^{\text {er }}$ que commence une nouvelle époque: la création de la Troisième section de la chancellerie privée de Sa Majesté comme police politique est une suite de la révolte des décembristes. Elle montre que la monarchie a résilié son alliance avec la noblesse, soupçonnée désormais de conspiration, tandis que le «peuple ", imbu du mythe du «bon tsar», est jugé de moins en moins capable de mener des actions politiques indépendantes. Ce sont les recherches de Pouchkine sur le soulèvement de Pugačev qui, entre autres causes, ont contribué à introduire le concept du monarchisme naïf du moujik russe, concept répété et renforcé par des générations d'historiens et qui jusqu'à ce jour détermine pour une large part l'image de la société russe à l'époque moderne.

Selon Rustemeyer cette image défigure l'univers mental des couches sociales inférieures, tel qu'il se manifeste à travers leurs prises de position dans les procès de slovo $i$ delo. Le rôle des faux tsars (samozvancy) comme porteurs des espoirs populaires a été surestimé. Loin de colporter des légendes et des rumeurs sans fondement, le discours des accusés révèle une rationalité croissante. Un bon exemple en est l'imputation des mauvaises récoltes au mauvais tsar (surtout à Pierre $\mathrm{I}^{\mathrm{er}}$ comme "Antéchrist »), habituellement interprétée comme preuve du mysticisme de la paysannerie russe. À y regarder de plus près, on découvre en arrière-plan des arguments tout à fait rationnels : ce sont les levées de recrues, dont le tsar est rendu responsable, qui enlèvent les laboureurs à leurs champs et désorganisent l'agriculture. Par ailleurs, les procès montrent à quel point les Russes des couches inférieures étaient bien informés sur la vie et la politique du tsar, réformes comprises.

12 Rustemeyer en déduit que ce qui a été poursuivi par l'autorité comme dissidence témoigne plutôt d'un consensus, voire d'une participation de la population au processus de modernisation. Comptetenu de l'inefficacité des procès pour slovo $i$ delo comme instrument du contrôle social, c'est la relative stabilité sociale et politique de l'État qui est remarquable. Stabilité, pour Rustemeyer, ne veut pas dire que la population se bornerait à faire écho aux mesures et mots d'ordre lancés d'en haut; elle insiste au contraire sur le dynamisme du discours populaire qui ferait, selon elle, partie intégrante des efforts de modernisation.

Ce dynamisme, toutefois, est limité par la faiblesse ou la quasi-absence d'une sphère publique délibérative. L'auteur s'appuie sur une définition de Schmoeckel, selon laquelle l'espace public au bas Moyen Âge et à l'époque moderne est une sphère de " communication et d'interaction, qui relie comportements individuels et communauté par l'intermédiaire de l'honneur ». Rustemeyer reconnait qu'en Russie le recours au crime de lèse-majesté lors des nombreux conflits d'honneur a pour effet de dessaisir la communauté en transférant aux autorités la fonction d'arbitre de l'honneur. C'est là une différence considérable par rapport à l'Europe centrale et occidentale de la même époque. La plainte adressée aux autorités commence toujours, il est vrai, par citer les propos tenus par les délinquants présumés. Mais leur discours n'entre pas pour autant dans la sphère publique. Sans doute ne s'agit-il pas de "discours isolés"; mais les qualifier de "fragments d'information circulante» ne suffit pas à en faire des éléments 
de communication "publique»; la catégorie des "rumeurs» me semblerait ici plus appropriée.

Que l'on soit d'accord ou non avec l'interprétation révisionniste de l'auteur, son livre courageux, qui fait le lien entre l'analyse minutieuse d'une source unique et extrêmement riche, au niveau de la micro-histoire, et les processus de la grande Histoire, ouvre des perspectives inédites et fascinantes sur les mentalités et les pratiques des différentes couches sociales. Il nous montre en même temps une Russie entrelacée de façon complexe dans l'histoire de l'Europe. Il ne reste qu'à en recommander vivement la lecture.

\section{NOTES}

1. Terme introduit en 1933 par Theodor Mayer, et qui désigne une société fondée sur les liens de dépendance.

2. Le concept de la Sozialdisziplinierung (le fait de discipliner la société) a été développé par Gerhard Oestreich dans les années 1960. Il a eu un impact croissant sur la pratique de l'histoire sociale en Allemagne et a fortement contribué à l'apparition d'études sur la criminalité, dont la revue Crime, Histoire et Sociétés est l'équivalent à l'échelle internationale. Même si l'approche se veut universelle, les études présentées jusqu'ici gravitent généralement autour du bas Moyen Âge et de l'époque moderne, ce qui est sans doute dû à l'accent mis sur la constitution de l'État moderne.

3. D'une tout autre envergure que les « Justiciers » (sing. sudebnik) de 1497 et de 1550. 